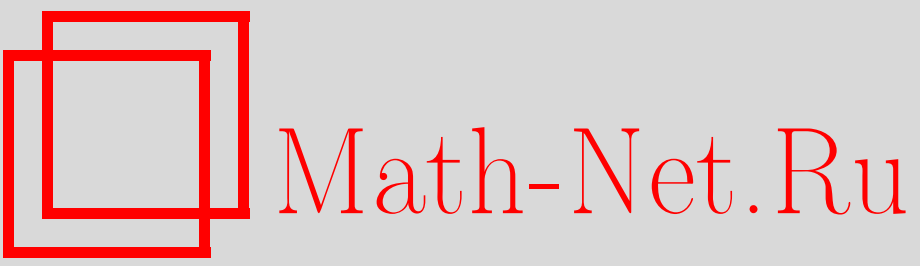

А. С. Черный, Качественное поведение решений стохастических дифференциальных уравнений с сингулярными коэффициентами, УМH, 2000, том 55, выпуск 3, 193-194

DOI: https://doi.org/10.4213/rm308

Использование Общероссийского математического портала Math-Net.Ru подразумевает, что вы прочитали и согласны с пользовательским соглашением

http://www.mathnet.ru/rus/agreement

Параметры загрузки:

IP: 3.85 .183 .62

26 апреля 2023 г., 17:21:05 


\title{
КАЧЕСТВЕННОЕ ПОВЕДЕНИЕ РЕШЕНИЙ СТОХАСТИЧЕСКИХ ДИФФЕРЕНЦИАЛЬНЫХ УРАВНЕНИЙ С СИНГУЛЯРНЫМИ КОЭФФИЦИЕНТАМИ
}

\author{
А. С. ЧЕРный
}

В настоящей статье рассматриваются одномерные однородные стохастические дифференциальные уравнения вида

$$
d X_{t}=b\left(X_{t}\right) d t+\sigma\left(X_{t}\right) d W_{t}, \quad X_{0}=x .
$$

Коэффициенты $b$ и $\sigma$ являются измеримыми функциями; никаких условий типа ограниченности или отделенности от нуля не предполагается. Единственное условие, налагаемое с самого начала, состоит в том, что $\forall x \in \mathbb{R}, \sigma(x) \neq 0$. Решение уравнения (1) будет пониматься в слабом смысле.

В статье [1] доказано, что если функция $(1+|b|) / \sigma^{2}$ локально интегрируема на $\mathbb{R}$, то для любого $x$ существует единственное решение уравнения (1). Цель настоящей статьи состоит в исследовании поведения решения вблизи тех точек, где функция $(1+|b|) / \sigma^{2}$ не локально интегрируема.

ОПРЕДЕЛЕниЕ 1. (а) Точка $d$ назьвается особой для уравнения (1), если функция $(1+|b|) / \sigma^{2}$ не локально интегрируема в этой точке.

(b) Точка $d$ называется изолированной особой точкой для уравнения (1), если $d$ - особая точка и существует проколотая окрестность $d$, состоящая из неособых точек.

ОПредЕление 2. (а) Пусть $T$ - момент остановки на $\left(\mathscr{F}_{t}\right)$, где $\left(\mathscr{F}_{t}\right)$ обозначает каноническую фильтрацию на пространстве $C\left(\mathbb{R}_{+}\right)$. Решением уравнения (1) до момента $T$ называется мера $\mathrm{P}$ на $\mathscr{F}_{T}$ такая, что

i) $\mathrm{P}\left\{X_{0}=x\right\}=1$ (здесь $X-$ координатный процесс на $C\left(\mathbb{R}_{+}\right)$);

ii) $\int_{0}^{T}\left(1+\left|b\left(X_{s}\right)\right|+\sigma^{2}\left(X_{s}\right)\right) d s<\infty$ Р-п.н.;

iii) процесс $M_{t}=X_{t}-\int_{0}^{t \wedge T} b\left(X_{s}\right) d s$ является $(\mathscr{F} t, \mathrm{P})$-локальным мартингалом с квадратической ковариацией $\langle M\rangle_{t}=\int_{0}^{t \wedge T} \sigma^{2}\left(X_{s}\right) d s$.

(b) Пусть $T$ - предсказуемый момент с предвещающей последовательностью $\left(T_{n}\right)_{n=1}^{\infty}$ (по поводу терминологии см. [2; гл. I]). Решением (1) до $T$ - называется мера Р на $\mathscr{F} T$ - такая, что $\forall n \in \mathbb{N}, \mathrm{P} \mid \mathscr{F}_{T_{n}}$ - решение (1) до $T_{n}$.

Пусть $I$ - некоторый интервал и $\left(\mathrm{P}_{x}\right)_{x \in I}-$ непрерьвный строго марковский процесс. Поведение этого процесса в правой полуокрестности выделенной точки $d \in I$ может быть охарактеризовано следующими параметрами:

$$
e_{1}=\lim _{b \downarrow d} \mathrm{P}_{d}\left\{T_{b}<\theta\right\}, \quad e_{2}=\lim _{c \downarrow d b \downarrow d} \lim _{b \downarrow} \mathrm{P}_{b}\left\{T_{c}<\theta\right\}, \quad e_{3}=\lim _{b \downarrow d} \mathrm{P}_{b}\left\{T_{d}<\theta\right\},
$$

где $\theta>0$ - некоторая константа. В [3; $\S 3.3]$ доказано, что значения параметров $e_{1}, e_{2}, e_{3}$ не зависят от выбора $\theta$. K примеру, значение $e_{3}=0$ показывает, что процесс не может достичь точку $d$ справа.

Предположим, что ноль - изолированная особая точка уравнения (1). Будем исследовать поведение решения в правой полуокрестности нуля. Установлено, что существует 8 различных типов поведения. Сформулируем для примера теорему, относящуюся к тому случаю, когда ноль имеет прав й тй 7 . Теоремы, относящиеся к остальным 7 случаям, схематично представлены на диаграмме. Мы используем следующие обозначения: $T_{0, a}=T_{0} \wedge T_{a}, \rho(x)=\exp \left\{-\int^{x} \frac{2 b}{\sigma^{2}}\right\}$, $s(x)=\int^{x} \rho$ (в случае, когда $\int_{0}^{a} \rho<\infty$, выбираем $s$ так, что $\left.s(0)=0\right)$. Здесь $a$ - такое положительное число, что функция $(1+|b|) / \sigma^{2}$ локально интегрируема на $(0, a]$ (т.е. локально интегрируема в каждой точке этого полуинтервала). 
Теорема. Предположим, что

$$
\int_{0}^{a} \rho(x) d x=\infty, \quad \int_{0}^{a} \frac{1+|b(x)|}{\rho(x) \sigma^{2}(x)}|s| d x=\infty, \quad \int_{0}^{a} \frac{|s(x)|}{\rho(x) \sigma^{2}(x)} d x<\infty .
$$

Для любого $х$ из интервала $I=(0, a]$ существует единственное решение уравнения (1), определенное до момента $T=T_{a}$. Зададим $\widetilde{\mathrm{P}}_{x}$ как образ $\mathrm{P}_{x}$ при отображении

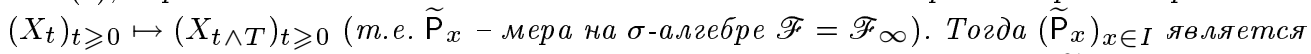
регулярным непрерывным строго марковским прочессом. Определим $\widetilde{\mathrm{P}}_{0}$ как меру, сосредоточенную на $X \equiv 0$. Тогда $\left(\widetilde{\mathrm{P}}_{x}\right)_{x \in[0, a]}$ - непрерывный строго марковский прочесс, для которого локальные характеристики в нуле имеют следующие значения: $e_{1}=0$, $e_{2}=1, e_{3}=0$.

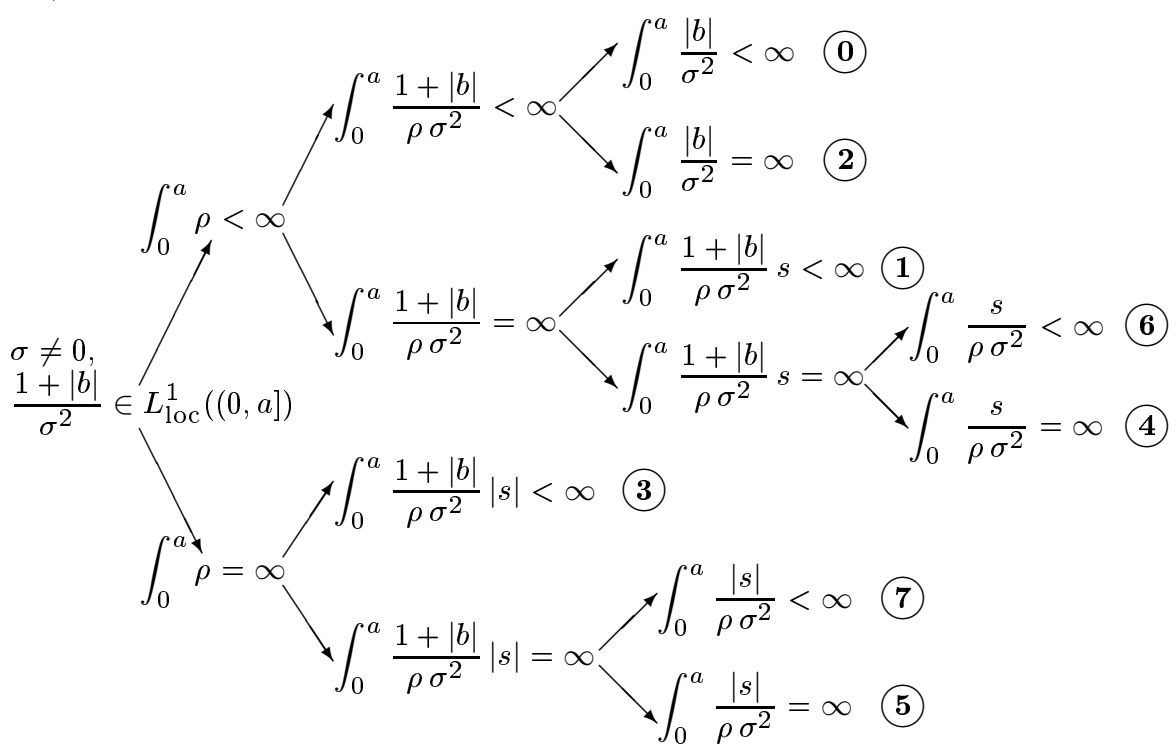

\begin{tabular}{|c|c|c|c|c|c|c|c|c|}
\hline Тип & $\mathbf{0}$ & $\mathbf{1}$ & $\mathbf{2}$ & $\mathbf{3}$ & $\mathbf{4}$ & $\mathbf{5}$ & $\mathbf{6}$ & $\mathbf{7}$ \\
\hline$I$ & {$[0, a]$} & {$[0, a]$} & {$[0, a]$} & {$[0, a]$} & $(0, a)$ & $(0, a]$ & $(0, a)$ & $(0, a]$ \\
\hline$T$ & $T_{0, a}$ & $T_{0, a}$ & $T_{a}$ & $T_{a}$ & $T_{a}-$ & $T_{a}$ & $T_{0, a}-$ & $T_{a}$ \\
\hline$e_{1}$ & 0 & 0 & 1 & 1 & 0 & 0 & 0 & 0 \\
\hline$e_{2}$ & 0 & 0 & 1 & 1 & 0 & 0 & 0 & 1 \\
\hline$e_{3}$ & 1 & 1 & 1 & 0 & 0 & 0 & 0 & 0 \\
\hline
\end{tabular}

Автор благодарит профессора А.Н. Ширяева за полезные обсуждения и ценные рекомендации.

\section{СПИСОК ЛИТЕРАТУРЫ}

[1] 1991. V. 151. Р. 149-197. [2] ЖКакод ЖК., Ширяев А. Н. Предельные теоремы для случайных процессов. М.: Физматлит, 1994. [3] Ито К., Маккин Г. Диффузионные процессы и их траектории. М.: Мир, 1968. 